\title{
Usages Socioeconomiques Des Espèces Ligneuses Au Sahel: Cas De Guidan Roumdji Au Niger
}

\section{Laminou M. Ousmane, (Maitre-assistant, Forestier - Environnementaliste)}

Université Dan Dicko Dankoulodo de Maradi, Faculté d'Agronomie et des Sciences de l'Environnement, Département de Génie Rural et Eaux \& Forêts. Maradi, Niger Boubé Morou, (Maitre de Conférences, Botaniste-Ecologue)
Saley Karim, (Maitre-assistant, Botaniste)
Oumarou Bachir Garba, (Doctorant, Ecologue)

Université Dan Dicko Dankoulodo de Maradi,

Faculté des Sciences et Techniques, Département de Biologie. Maradi, Niger Ali Mahamane, (Professeur Titulaire, Botaniste-Ecologue) Université de Diffa, Faculté des Sciences et Techniques, Département de Biologie. Diffa, Niger

doi: 10.19044/esj.2017.v13n26p355 URL:http://dx.doi.org/10.19044/esj.2017.v13n26p355

Abstract

Woody species are of multiple use in the department of GuidanRoumdji, in Niger. The present study carried out in Garin-yahaya and Tsayin-daka aims to identify the woody species, their different uses and to determine their present state. Data collection methodology consisted of a floristic inventory at the level of 80 plots and ethnobotanical surveys on a sample of 250 respondents. 46 species, divided into 38 genera and 22 families, were recorded. There were 24 species used in food, 19 fodder species and 38 others species used in the treatment of several ailments in the area. Also, in handicrafts and construction, a dozen species are used respectively against 19 others species in the production of wood energy. These diverse and wide-ranging uses, associated with the effects of climate change, affect the woody species populations. Thus, in the area, 25 species are declared extinct, 18 and 14 others are respectively considered threatened and rare. The study also reported on woody species management practices in the area, including the conservation of 7 local species and the introduction of 11 exotic species because of various products and services they provide. Also, 96\% of the local population practices the trees' farming natural regeneration management (FNRM) in their farms for various reasons. This 
would be a start in the sustainable and rational management of forest resources in the area with the maintenance of several woody species in the area.

Keywords: Woody species, uses, state, management, Niger

\section{Résumé}

Les espèces ligneuses sont d'un usage multiple dans le département de Guidan-Roumdji au Niger. La présente étude conduite à Garin-yahaya et Tsayin-daka vise à inventorier les espèces ligneuses à usages multiples, leurs différentes utilisations et apprécier leur état actuel. La méthodologie de collecte des données a consisté à un inventaire floristique au niveau de 80 placettes et des enquêtes ethnobotaniques sur un échantillon de 250 répondants. 46 espèces, reparties en 38 genres et 22 familles, ont été recensées. Il a été dénombré 24 espèces utilisées dans l'alimentation humaine, 19 espèces fourragères et 38 autres dans le traitement de plusieurs maux. Dans l'artisanat et dans la construction, 12 espèces sont utilisées et 19 autres dans la production du bois énergie. Ces utilisations diverses et très variées, associées aux effets du changement climatique affectent les populations de ces espèces ligneuses. Ainsi, dans la zone, 25 espèces sont déclarées disparues, 18 et 14 autres sont considérées respectivement menacées et rares. Aussi, l'étude a rapporté des pratiques locales de gestion des espèces ligneuses dans la zone, notamment des actions de conservation de 7 espèces locales et d'introduction de 11 espèces exotiques pour plusieurs produits et services qu'elles fournissent. Egalement, 96\% de la population locale pratique la régénération naturelle assistée (RNA) des ligneux dans leurs champs pour diverses raisons. Ce qui constituerait une amorce dans la gestion durable et rationnelle des ressources forestières dans la zone avec le maintien des plusieurs espèces ligneuses dans la zone.

Mots-clés : Espèces ligneuses, usages, état, gestion, Niger

\section{Introduction}

En Afrique subsaharienne, les espèces ligneuses sont d'une très grande importance pour les populations, particulièrement rurales, en raison de leur utilisation dans la satisfaction des besoins en alimentation humaine et animale, en pharmacopée, en énergie et services aussi bien dans la génération des revenus et autres aspects du bien-être de l'homme (Oumorou et al., 2010 ; Fandohan, 2011 ; Zerbo et al., 2011 ; Dan Guimbo et al., 2012 ; Thiombiano et al., 2012). Ceci est illustré par les nombreux produits et services forestiers non ligneux (feuilles, fleurs, fruits, graines, écorces, sèves, fibres, rhizomes, etc.) fournis par les ligneux à usages multiples (FAO, 
2001 ; Tieguhong et al., 2009 ; Achigan-Dako et al., 2010 ; Zerbo et al., 2011). Ainsi, on dénombre plusieurs ligneux des forêts tropicales dont toutes les parties ou presque sont quotidiennement utilisées en médecine traditionnelle mais aussi comme bois d'œuvre ou bois de service (Fandohan et al., 2008). Cependant, ces ligneux et leurs habitats subissent des perturbations, liées à ces actions anthropiques et aux changements climatiques, qui menacent leur survie alors même que leurs caractérisations écologiques, morphologiques, génétiques et l'inventaire de leur utilité n'aient été approfondies (Natta et al., 2011). Il existe même plusieurs exemples d'essences ligneuses menacées du fait d'une surexploitation (Sinsin et al., 2004 ; Glélé-Kakaï et al., 2009). En effet, ces dernières décennies, l'Afrique a été caractérisée par un taux annuel de déforestation de l'ordre de $0,7 \%$, soit plus du double de la moyenne mondiale (Yaméogo et al., 2009). La diminution des ressources ligneuses affecte tous les domaines agroécologiques de la vie en milieu rural. Ainsi, au Sahel, la situation se caractérise par une dégradation accélérée des sols déjà fragilisés par nombre d'aléas, une production agricole et pastorale insuffisante, une insécurité alimentaire de plus en plus dramatique et une population en croissance rapide (Boubacar, 2010).

Au Niger, la population est estimée, en 2016, à 20283950 habitants avec un taux d'accroissement naturel de 3,9\% (INS, 2013). Près de $84 \%$ de celle-ci vit en milieu rural et tire l'essentiel des moyens de subsistance de l'exploitation des ressources naturelles (Baggnian, 2010). Ainsi, les ligneux constituent des apports importants dans l'alimentation humaine et animale pour environ 50 à $70 \%$ des besoins, dans la pharmacopée traditionnelle pour 80 à $90 \%$, dans l'énergie et la construction pour 90\% (Sani, 2009). Il est évident que le maintien de ces espèces ligneuses utiles pour les populations passe par la conservation des peuplements ligneux à travers une gestion rationnelle et durable. Cette conservation reste plus urgente dans la zone sahélienne où les espèces végétales se raréfient rapidement. La mise en œuvre de celle-ci, dans le contexte de dégradation des ressources naturelles que vit cette région, s'avère nécessaire et passe par une meilleure connaissance des espèces ligneuses en place et leur typologie d'utilisations, leur état et les pratiques de gestion de ces ressources. Cette étude s'inscrit dans ce cadre dont l'objectif est de contribuer à l'identification des usages des ligneux dans les terroirs de Garin Yahaya et Tsayin Daka dans le département de Guidan-Roumdji au centre du Niger.

\section{Matériel et méthodes \\ Sites d'étude}

Les terroirs de Garin-yahaya et de Tsayin-Daka sont situés dans le département de Guidan Roumdji (Région du Maradi) au Centre-sud du 
Niger, en zone sahélienne. Ce département est compris entre la latitude 13'39'31', Nord et la longitude 06² $41^{\prime} 57^{\prime}$ ' Est. Localisés dans la zone climatique semi-aride, les sites d'étude présentent une végétation de type savane arbustive avec un cumul pluviométrique annuel de 437,9 $\mathrm{mm}$ en moyenne sur les 30 dernières années, des températures minimales fluctuant entre 15 et $20^{\circ} \mathrm{C}$ pendant la saison sèche froide (décembre - février) et des maximales atteignent 39 à $42^{\circ} \mathrm{C}$ pendant la saison sèche chaude (avril - juin) (Issa et Oumarou, 2006). Les sols sableux sont les plus dominants et sont de faible fertilité. Ils sont pauvres en matières organiques à cause de l'érosion hydrique et éolienne, de l'absence de jachère et du défrichement abusif (Anonyme, 2013). Ils constituent le terrain de prédilection des cultures telles que le mil, l'arachide et le niébé.

\section{Collecte de données}

Dans le cadre de l'inventaire pour la détermination de la flore ligneuse, la méthode d'échantillonnage stratifié a été utilisée. Il comporte deux niveaux de stratification : Le niveau d'occupation des sols et celui de la pratique de la régénération naturelle assistée (RNA). Le premier niveau a consisté à faire un échantillonnage à l'intérieur de 3 types d'occupation des sols (aires de pâturage, champs de cultures et couloirs de passage). A l'intérieur de chacun de ces types d'occupation des sols, des relevés ont été réalisés à des distances aléatoires suivant un transect qui va du village à la brousse et tenant compte de la densité des ligneux. Le deuxième niveau a consisté à considérer un échantillonnage à l'intérieur des champs avec RNA et champs sans RNA. A ce niveau aussi, les relevés ont été réalisés à des distances aléatoires suivant un transect allant du village à la brousse. La collecte des données a été réalisée dans 41 placettes carrées de $50 \mathrm{~m}$ x $50 \mathrm{~m}$ dans les champs et 39 placettes rectangulaires de $50 \mathrm{~m}$ x $20 \mathrm{~m}$ dans les aires de pâturages et couloirs de passage d'animaux, soit un total de 80 placettes inventoriées sur les deux sites. Dans chacune des placettes, les noms de toutes les espèces ligneuses présentes ont été enregistrés.

Dans la collecte des données ethnobotaniques, les informations ont été obtenues à travers des enquêtes au moyen de questionnaires préalablement élaborés. Les informations recueillies portent sur les espèces disparues, les espèces menacées, les espèces rares, les espèces conservées, les espèces introduites, la pratique de la RNA et les différentes utilisations des ligneux. Un échantillon de 250 personnes au total ont été enquêtées dont 200 hommes et 50 femmes, soit 100 hommes et 25 femmes au niveau de chacun des deux villages. En plus du genre, le choix des enquêtés a été aussi fonction des activités socioprofessionnelles (agriculteurs, artisans, tradipraticiens, éleveurs, exploitants de bois, revendeurs de bois) et de classes d'âge. La classe de 54 à 65 ans a été la plus sollicitée par les enquêtes 
(32\%) (elle détient plus des connaissances ethnobotaniques que les jeunes), suivie de celles de 24 à 35 ans (24\%), de 34 à 45 ans (22\%) et de 44 à 55 ans $(22 \%)$. Le fort pourcentage d'hommes $(80 \%)$ est dû à la disponibilité de ces derniers par rapport aux femmes pendant la phase de collecte des données (mois de septembre et d'octobre) mais aussi à l'exercice quasi masculin des activités socioprofessionnelles prises en compte dans l'échantillonnage.

\section{Traitement et analyse des données}

Les données quantitatives collectées ont été dépouillées et saisies à l'aide du tableur de traitement de données Microsoft Office Excel 2007.

Le logiciel SPSS (version 17.0) a été utilisé pour les analyses statistiques des données issues des enquêtes. Il a été utilisé dans le calcul des fréquences.

\section{Résultats}

\section{Caractéristiques floristiques}

L'analyse taxonomique révèle une richesse spécifique de 46 espèces de ligneux reparties en 22 familles et 38 genres.

L'analyse de l'importance numérique des familles de la liste floristique (Tableau 1) montre que les familles les mieux représentées en nombre d'espèces sont les Mimosaceae $(21,74 \%)$, les Caesalpiniaceae $(13,04 \%)$, les Combretaceae $(8,70 \%)$, les Anacardiaceae et les Capparaceae $(6,52 \%$ chacune). Les familles les moins représentées sont entre autres les Sterculiaceae et les Verbenaceae (2,17\% chacune).

Tableau 1: Nombre d'espèces et de genres par famille

\begin{tabular}{ccccc}
\hline Familles & $\begin{array}{c}\text { Nombre } \\
\text { d'espèces }\end{array}$ & $\begin{array}{c}\text { \% } \\
\text { d'espèces }\end{array}$ & Nombre de genres & $\begin{array}{c}\text { \% de } \\
\text { genres }\end{array}$ \\
\hline Mimosaceae & 10 & 21,74 & 6 & 15,79 \\
Caesalpiniaceae & 6 & 13,04 & 5 & 13,16 \\
Combretaceae & 4 & 8,7 & 3 & 7,89 \\
Anacardiaceae & 3 & 6,52 & 3 & 7,89 \\
Capparaceae & 3 & 6,52 & 2 & 5,26 \\
Burseraceae & 2 & 4,35 & 2 & 5,26 \\
Euphorbiaceae & 2 & 4,35 & 2 & 5,26 \\
Tiliaceae & 2 & 4,35 & 1 & 2,63 \\
Annonaceae & 1 & 2,17 & 1 & 2,63 \\
Arecaceae & 1 & 2,17 & 1 & 2,63 \\
Asclepiadaceae & 1 & 2,17 & 1 & 2,63 \\
Balanitaceae & 1 & 2,17 & 1 & 2,63 \\
Bignoniaceae & 1 & 2,17 & 1 & 2,63 \\
Bombacaceae & 1 & 2,17 & 1 & 2,63 \\
Chrysobalanaceae & 1 & 2,17 & 1 & 2,63 \\
Ebenaceae & 1 & 2,17 & 1 & 2,63 \\
Loganiaceae & 1 & 2,17 & 1 & 2,63
\end{tabular}




\begin{tabular}{ccccc} 
Meliaceae & 1 & 2,17 & 1 & 2,63 \\
Rhamnaceae & 1 & 2,17 & 1 & 2,63 \\
Sapotaceae & 1 & 2,17 & 1 & 2,63 \\
Sterculiaceae & 1 & 2,17 & 1 & 2,63 \\
Verbenaceae & 1 & 2,17 & 1 & 2,63 \\
\hline Total & $\mathbf{4 6}$ & $\mathbf{1 0 0}$ & $\mathbf{3 8}$ & $\mathbf{1 0 0}$ \\
\hline
\end{tabular}

\section{Aspects ethnobotaniques et état des espèces ligneuses}

Les résultats des enquêtes ethnobotaniques ont révélé six différents types d'utilisation des ligneux dans la zone d'étude: alimentation humaine et animale, pharmacopée, artisanat, énergie et construction.

C'est ainsi que 24 espèces ligneuses alimentaires ont été recensées (Tableau 2). Les parties de la plante utilisées sont les feuilles, les fruits et la gomme.

Tableau 2. Espèces ligneuses utilisées dans l'alimentation humaine et les différentes parties et modes d'utilisation

\begin{tabular}{lcc}
\hline \multicolumn{1}{c}{ Espèces } & $\begin{array}{c}\text { Parties } \\
\text { consommées }\end{array}$ & Modes de consommation \\
\hline Adansonia digitata & Feuilles, fruits & Sauce, consommés crus \\
Annona senegalensis & Fruits & Consommés crus \\
Balanites aegyptiaca & Fruits & Consommés crus \\
Borassus aethiopum & Fruits & Consommés crus \\
Boscia senegalensis & Fruits & Consommés crus \\
Combretum micranthum & Fruits & Consommés crus \\
Combretum nigricans & Gomme & Comestible \\
Detarium microcarpum & Fruits & Consommés crus \\
Diospyros mespiliformis & Fruits & Consommés crus \\
Grewia bicolor & Fruit & Consommé cru \\
Isoberlinia doka & Feuilles & Sauce \\
Lannea microcarpa & Fruit & Consommé cru \\
Moringa oleifera & Feuilles & Comestible \\
Neocarya macrophylla & Fruit & Consommé cru \\
Parkia biglobosa & Fruit & Consommé cru, soumbala \\
Prosopis africana & Fruit & Consommé cru, soumbala \\
\multicolumn{1}{c}{ Sclerocarya birrea } & Feuilles, fruit & Consommé cru \\
Sterculia setigera & Fleur & Sauce \\
Tamarindus indica & Fruit & Jus \\
Vitellaria paradoxa & Fruit & Consommé cru, jus \\
Vitex doniana & Fruit & Consommé cru \\
Ximenia americana & Fruit & Consommé cru \\
Ziziphus mauritiana & Fruit & Consommé cru \\
Ziziphus spina-christi & Fruit & Consommé cru \\
\hline
\end{tabular}


Les espèces ligneuses sont aussi connues pour être utilisées comme sources de fourrages dans la zone pendant la saison sèche. Les résultats de l'étude ont ainsi révélé que 19 espèces ligneuses ont été recensées pour cet usage où leurs feuilles et fruits rentrent dans l'alimentation du bétail (Tableau 3).

Tableau 3. Espèces ligneuses fourragères et les différentes parties et modes d'utilisation

\begin{tabular}{ccc}
\hline Espèces & Parties utilisées & Modes d'utilisation \\
\hline Acacia nilotica & Feuilles & Brouté \\
Acacia seyal & Feuilles & Brouté \\
Acacia tortilis & Feuilles, fruit & Brouté \\
Albizia chevalieri & Feuilles & Brouté \\
Balanites aegyptiaca & Feuilles & Brouté \\
Boscia senegalensis & Feuilles & Brouté \\
Combretum glutinosum & Feuilles & Brouté \\
Combretum micranthum & Feuilles & Brouté \\
Entada africana & Feuilles & Brouté \\
Faidherbia albida & Feuilles, fruit & Brouté \\
Grewia bicolor & Feuilles & Brouté \\
Guiera senegalensis & Feuilles & Brouté \\
Lannea microcarpa & Feuilles & Brouté \\
Piliostigma reticulatum & Feuilles, fruit & Brouté \\
Sclerocarya birrea & Feuilles & Brouté \\
Sterculia setigera & Feuilles & Brouté \\
Ziziphus mauritiana & Feuilles & Brouté \\
Ziziphus spina-christi & Feuilles & Brouté \\
\hline
\end{tabular}

Dans la pharmacopée traditionnelle, quelques 38 espèces ligneuses ont été recensées comme utilisées dans le traitement de plusieurs pathologies et autres maladies ou infections (Tableau 4). Les parties de la plante utilisées dans la zone d'étude regroupent l'écorce, les feuilles, les fruits et les racines.

Tableau 4. Espèces ligneuses, différentes parties utilisées et principaux maux et maladies traités en pharmacopée traditionnelle

\begin{tabular}{ccl}
\hline Espèces & Parties utilisées & $\begin{array}{l}\text { Principales maladies } \\
\text { et symptômes traités }\end{array}$ \\
\hline Acacia nilotica & Ecorce, feuilles, fruit & Plaie, hémorragie, infections \\
Acacia senegal & Ecorce & Sorcellerie \\
Albizia chevalieri & Ecorce, feuilles & Toux, dysenterie \\
Anacardium occidentale & Ecorce, feuilles, racines & Diabète, toux, dysenterie \\
Anogeissus leiocarpa & Feuilles & Déparasitage \\
Azadirachta indica & Ecore, feuilles & Hémorroïde, chaleur du corps \\
Balanites aegyptiaca & Ecorce, feuilles, racines & Maux de l'œil, ventre \\
Boscia angustifolia & Ecorce & Maux de ventre \\
Boscia salicifolia & Ecorce, racines & Hémorroïde, excitant
\end{tabular}


Boscia senegalensis

Cassia singueana

Cassia sieberiana

Combretum glutinosum

Combretum micranthum

Combretum nigricans

Diospyros mespiliformis

Ficus polita

Ficus thonningii

Grewia bicolor

Grewia flavescens

Guiera senegalensis

Khaya senegalensis

Lannea microcarpa

Piliostigma reticulatum

Prosopis africana

Pterocarpus erinaceus

Sclerocrya birrea

Securidaca longipedunculata

Sterculia setigera

Stereospermum kunthianum

Tamarindus indica

Terminalia avicennioides

Vitellaria paradoxa

Ximenia americana

Ziziphus mauritiana
Feuilles

Racines

Ecorce, racines

Feuilles, racines

Feuilles

Ecorce

Ecorce, feuilles

Ecorce, feuilles, racines

Ecorce, feuilles, racines

Ecorce, feuilles, racines

Ecorce, fruit, racines

Feuilles, racines

Ecorce, racine

Ecorce, feuilles

Ecorce, feuilles

Ecorce, feuilles, racines

Ecorce, feuilles, racines

Ecorce, racines

Ecorce, feuilles, racines

Ecorce, feuilles, racines

Ecorce, feuilles, racines

Ecorce, feuilles, racines

Ecorce, racines

Ecorce, fruit, racines

Ecorce, feuilles, racines

Ecorce, feuilles, racines
Maux de ventre, l'œil, rhume

problème pulmonaire

Hémorroïde

Toux, rhume, vomissement

Tétanos

Maux de ventre, rhume

Maux de tête, œdème, folie

Hémorroïde, malnutrition

Jaunisse, choléra

toux, venin, fatigue

Maux du cœur, fatigue, aphrodisiaque

Maux de ventre, toux, rhume

Hémorroïde

Hémorroïde, plaie, stérilité

Maux de ventre, tête, ventre

Brûlure, dermatose, vertige

Plaie, toux, maladies mentales

Dysenterie, hémorroïde

Force physique

Paludisme, toux,

Plaie, brûlure, asthme

Paralysie, plaie, stérilité, entorse œdème, ulcère, diarrhée, hémorrö̈de Pédicure, bilharziose, cancer du foie Sevrage, tuberculose, angine, asthme Maux de ventre, sorcellerie

Dans l'artisanat aussi, les espèces ligneuses sont utilisées dans la fabrication d'objets divers. C'est ainsi que 12 espèces ligneuses utilisées dans l'artisanat ont été recensées (Tableau 5). Les parties prélevées concernent le tronc, les branches, les feuilles et les fruits.

Tableau 5. Différentes parties et usages des espèces ligneuses en artisanat

\begin{tabular}{ccc}
\hline Espèces & Parties utilisées & Usages \\
\hline Acacia nilotica & Tronc, fruit & Manches (outils), tanin \\
Acacia senegal & Tronc, branches & Manches (outils) \\
Annona senegalensis & Tronc, branches & Manches (outils) \\
Balanites aegyptiaca & Tronc, branches & Manches (outils) \\
Combretum micranthum & Tronc, branches & Lit, tabouret, bâton \\
Diospyros mespiliformis & Tronc, branches & Manches (outils) \\
Grewia bicolor & Tronc & Manches (outils), pilon \\
Hyphaene thebaica & Feuilles & Natte, chapeau, corde \\
Lannea microcarpa & Tronc, branches & Mortier, pilon \\
Prosopis africana & Tronc, branches & Mortier, pilon \\
Sclerocarya birrea & Tronc, branches & Mortier, pilon \\
Ziziphus mauritiana & Tronc & Manches (outils), pilon \\
\hline
\end{tabular}


Dans les zones rurales, les espèces ligneuses jouent un rôle très important dans la satisfaction des besoins énergétiques. C'est ainsi que 19 espèces utilisées dans la production du bois énergie ont été recensées dans la zone d'étude (Tableau 6). Les principales parties utilisées sont le tronc, les branches et secondairement les rameaux et les écorces.

Tableau 6. Différentes parties utilisées et usages des espèces ligneuses dans bois-énergie

\begin{tabular}{ccl}
\hline Espèces & Parties utilisées & Usages \\
\hline Acacia nilotica & Tronc, branches, rameaux & Charbon, chauffe \\
Acacia tortilis & Tronc, branches, rameaux & Charbon, chauffe \\
Acacia senegal & Tronc, branches, rameaux & Charbon, chauffe \\
Albizia chevalieri & Tronc, branches, rameaux & Chauffe \\
Annona senegalensis & Tronc, branches, rameaux & Chauffe \\
Balanites aegyptiaca & Tronc, branches, rameaux & Charbon, chauffe \\
Cassia sangueana & Tronc, branches, rameaux & Chauffe \\
Cassia sieberiana & Tronc, branches, rameaux & Chauffe \\
Combretum glutinosum & Branches, rameaux & Chauffe \\
Combretum micranthum & Tronc, branches, rameaux & Chauffe \\
Dichrostachys cinerea & Tronc, branches, rameaux & Charbon, chauffe \\
Diospyros mespiliformis & Tronc, branches, rameaux & Charbon, chauffe \\
Entada africana & Branches, rameaux & Chauffe \\
Guiera senegalensis & Tronc, branches, rameaux & Chauffe \\
Lannea microcarpa & Branches, rameaux & Chauffe \\
Parkia biglobosa & Tronc, branches, rameaux & Chauffe \\
Piliostigma reticulatum & Branches, rameaux & Chauffe \\
Prosopis africana & Tronc, branches, rameaux & Charbon, chauffe \\
Sclerocarya birrea & Branches, rameaux & Chauffe \\
Stereospermum kunthianum & Tronc, branches, rameaux & Chauffe \\
\hline
\end{tabular}

Les populations de la zone d'étude utilisent aussi les produits des espèces ligneuses dans la construction. Ainsi, 12 espèces ont été recensées pour cet usage (Tableau 7). Les troncs, branches, rameaux des arbres sont les parties sollicitées.

Tableau 7. Différentes parties et usages des espèces ligneuses dans la construction

\begin{tabular}{ccc}
\hline Espèces & Parties utilisées & Usages \\
\hline Acacia nilotica & Tronc, branches & Toit, hangar, clôture, grenier, case \\
Acacia tortilis & Tronc, branches & Toit, hangar, clôture, grenier, case \\
Acacia senegal & Tronc, branches & Toit, hangar, clôture, grenier, case \\
Annona senegalensis & Tronc, branches & Toit, clôture, grenier, case \\
Azadirachta indica & Branches, rameaux & Toit, hangar, clôture \\
Calotropis procera & Tronc, branches & Toit, hangar, clôture, case \\
Combretum glutinosum & Branches, rameaux & Toit, hangar, clôture \\
Combretum micranthum & Tronc, branches & Toit, hangar, clôture, grenier, case \\
Guiera senegalensis & Tronc, branches & Toit, hangar, clôture, grenier, case
\end{tabular}




\begin{tabular}{ccc} 
Lannea microcarpa & Branches, rameaux & Toit, hangar, clôture \\
Piliostigma reticulatum & Branches, rameaux, écorce & Toit, hangar, clôture \\
Sclerocarya birrea & Branches, rameaux & Toit, hangar, clôture \\
\hline
\end{tabular}

\section{Etat des espèces ligneuses}

Les résultats des enquêtes ethnobotaniques sur l'état des peuplements d'espèces disparues, menacées, rares, conservées et introduites dans la zone d'étude (Annexe 1) se résument comme suit : 25 espèces ligneuses sont déclarées disparues par la population locale. Les causes de la disparition de ces espèces évoquées, par ordre d'importance, sont l'écorçage $(35,80 \%)$, la coupe illégale $(31,80 \%)$, le déracinement $(20,80 \%)$, l'effeuillage $(6,40 \%)$, les sécheresses $(3,1 \%)$ et enfin la cueillette $(2,1 \%)$.

Aussi, 18 espèces sont considérées comme menacées à cause des coupes illégales $(74,4 \%)$, de l'écorçage $(12 \%)$, de la mauvaise gestion $(7,90 \%)$, du déracinement $(2,90 \%)$, de l'effeuillage $(2,1 \%)$ et enfin des sécheresses $(0,80 \%)$. Egalement, 14 espèces ligneuses ont été déclarées rares à cause de la mauvaise gestion (70\%), de l'absence de leur plantation (20\%) et des sécheresses $(10 \%)$.

Par contre, il a été rapporté que certaines espèces ligneuses sont conservées par la population elle-même. La conservation de celles-ci, au nombre de 7 , est expliquée pour les besoins en leurs fruits $(25,7 \%)$, leurs feuilles $(21,9 \%)$, leur bois énergie $(17,9 \%)$, leurs écorces $(13,6 \%)$, leur ombre $(12,1 \%)$, leurs planches $(5,7 \%)$, la protection de l'environnement $(1,7 \%)$ et enfin leurs racines $(1,4 \%)$. Aussi, 11 espèces exotiques ont été introduites dans la zone pour les besoins de la diversification des espèces ligneuses (35\%), du reverdissement (30\%), de l'alimentation humaine (25\%) et de l'ombre $(10 \%)$.

Les résultats des enquêtes ethnobotaniques ont également révélé que $96 \%$ de la population locale a adopté la pratique de la régénération naturelle assistée (RNA) des ligneux dans les champs, une pratique qui participe à la promotion de la gestion durable des ressources naturelles. Les principales raisons de cette adoption sont l'entretien des espèces ligneuses $(34,4 \%)$, la protection contre les vents $(28 \%)$ et la fertilisation des champs $(26,4 \%)$. Les autres raisons de cette pratique de la RNA incluent la reconstitution du peuplement ligneux $(4,8 \%)$, l'utilisation des produits (bois, feuilles, fruits, fleur) issus de la RNA (4\%) et l'obtention de récompense auprès du projet PASADEM, initiateur de l'approche de la RNA dans la zone $(2,4 \%)$.

\section{Discussion}

L'analyse floristique montre que la zone d'étude compte 46 espèces ligneuses reparties entre 22 familles et 38 genres. Cette richesse floristique est bien supérieure à d'autres trouvées en zone sahélienne du Niger, à 
Mirriah (Baggnian et al., 2013) et à Dan Kada Dodo-Dan Gado dans le département d'Aguié (Abdourahamane et al., 2013), et du Sénégal, à Ranérou (Ndiaye et al., 2010). Ce pourrait s'expliquer, en partie, par le mode de dissémination de la majorité des espèces dominantes appartenant aux familles inventoriées. En effet, les Combretaceae sont caractérisées par leurs fruits ailés facilement disséminés par le vent alors que les Caesalpiniaceae, généralement fourragères, aux semences zoochores sont disséminées par les herbivores qui les consomment (Ouédraogo, 2009). Ces familles sont composées d'espèces qui résistent notamment au manque et à l'insuffisance des pluies, mais aussi aux fortes températures (Savadogo et al., 2016).

Cependant, le nombre des espèces alimentaires de la zone reste inférieur à celui recensé par Dan Guimbo et al. (2012) sur les zones périphériques du parc $\mathrm{W}$ du Niger. Cette différence est probablement liée à la zone d'étude qui constituent une large étendue caractérisée par une variété d'écosystèmes et une diversité d'espèces végétales (Mahamane, 2005 ; Inoussa, 2011). Néanmoins, ces espèces possèdent une bonne valeur nutritive et sont très appréciées par la population (Guigma et al., 2012).

La zone d'étude, bien qu'elle soit agricole, possède un nombre assez important d'espèces ligneuses fourragères (19 espèces) avec une valeur fourragère et très appréciées des animaux. Ces espèces constituent une source fourragère d'une grande importance surtout en saison sèche, période où les espèces herbacées sont rares. Leur nombre est supérieur à celui recensé par Sani (2009) dans le département de Mirriah. Cette différence pourrait s'expliquer par le fait que notre zone d'étude est spécifiquement plus riche. Cette importante proportion des ligneux fourragers est d'ailleurs conforme à la végétation de l'Afrique de l'Ouest où plus de $75 \%$ des arbres et arbustes sont fourragers (Kaboré-Zoungrouna, 1995).

Il a été recensé 36 espèces ligneuses utilisées dans la pharmacopée traditionnelle pour un large spectre d'action thérapeutique. Cependant, ce nombre reste inférieur à celui de Khossanto à kédougou au Sénégal (Oumar et al., 2014). Cela pourrait s'expliquer par la différence écologique plus humide de Khossanto où domine la savane arborée dense (climat soudanosahélien avec 800 à $1300 \mathrm{~mm}$ de pluies annuelles) et les diverses traits socioculturels de sa population où cohabitent quelques cinq ethnies (GERAD, 2010).

L'étude a aussi mis en évidence l'usage d'une douzaine espèces ligneuses dans l'artisanat et la construction. Elles fournissent un bois dur et solide résistant aux termites. En plus, leurs produits (tronc, branches, feuilles, rameaux et fruits) sont largement commercialisés et procurent des revenus non négligeables dans les foyers, généralement très pauvres. Néanmoins, ces usages sont en régression du fait de l'interdiction de 
l'exploitation de la plupart d'entre elles, classées comme espèces menacées par l'état du Niger.

Tous ces usages socioéconomiques par les différents acteurs entrainent des pressions très importantes sur ces ressources ligneuses de la zone et leur habitat. Ces différentes pressions anthropiques exercées sur les peuplements ont un impact négatif important, induisant une modification de leur structure et accentuant leur dégradation. Selon la population enquêtée, l'écorçage, la coupe illégale, le déracinement, l'effeuillage et la cueillette restent les principaux facteurs qui causent la disparition de plusieurs espèces ligneuses, Ainsi, ils ont causé la disparition de 25 espèces dans la zone. Il est évident que dans un pays où plus de $80 \%$ de la population vit en milieu rural, cette pression pourrait avoir de graves conséquences sur l'environnement (Hamadou, 2008 ; Sani, 2009). Aussi, d'autres espèces ligneuses (18) sont considérées comme menacées de disparition à cause de ces mêmes facteurs. Toutefois, les modes d'exploitation dans cette zone constituent une menace pour la plupart des espèces, surtout lorsque le feuillage de l'arbre se trouve hors de la portée des animaux. Dans ces cas-ci, les bergers n'hésitent pas à mutiler ou à abattre l'arbre (Sarr et al., 2013). Déjà, quelques 14 espèces sont considérées comme rares dans la zone d'étude à cause des prélèvements et de la mauvaise gestion des peuplements. La conséquence est qu'il en résulte une dégradation progressive de l'environnement qui se traduit par une raréfaction des espèces ligneuses (Devineau et Guillaumet, 1992). Même si les causes naturelles ont été davantage évoquées par la population, les causes anthropiques jouent également un rôle important dans la dégradation des ressources forestières (Dan Guimbo et al., 2010). En effet, les conditions climatiques difficiles des dernières décennies, la demande de plus en plus croissante en terres agricoles, la pression continue du bétail, l'inadéquation des pratiques de gestion, sont autant de facteurs qui ont réduit considérablement la présence des arbres, ressources essentielles dans le Sahel (Sarr et al., 2013).

Dans la zone, les paysans ont pris conscience de la nécessité de s'organiser pour résoudre les problèmes communs que sont la baisse de la production agricole, la pauvreté mais aussi de la dégradation de l'environnement (Dramé et Berti, 2008). Les résultats des enquêtes ont relevé que 7 espèces ligneuses, parmi les plus utilitaires et multifonctionnelles, font l'objet de conservation. 11 autres espèces ligneuses exotiques ont été introduites dans la zone pour des besoins de diversification, de reverdissement, d'alimentation humaine et de l'ombre.

Dans le même sens, la population a très largement adopté la pratique de la RNA dans les champs. Ce qui laisse suggérer une tendance à la promotion de la gestion durable des ressources naturelles. Avec cette large adoption de cette pratique de la régénération naturelle, il serait possible de 
couvrir les besoins des populations locales mais aussi d'assurer la conservation des espèces ligneuses. En effet, plus de 4,8 millions d'hectares sont plus verts de nos jours que deux décennies auparavant dans les régions de Zinder et de Maradi grâce, essentiellement, aux arbres de régénération naturelle gérés par les paysans dans les zones densément peuplées et surexploitées à des fins agricoles (Larwanou et al., 2006). La RNA a permis un changement de perception. En effet, les paysans ne considèrent plus l'arbre comme appartenant à l'Etat, mais comme une propriété privée. Et, tout exploitant qui prend un champ en gage, n'a aucun droit sur les ligneux qui s'y trouvent (Adamou, 2012).

\section{Conclusion et perspectives}

La présente étude conduite dans le département de Guidan Roumdji a permis de mettre en évidence la liste de la flore ligneuse utiles pour les populations de la zone. L'analyse de cette richesse spécifique a montré que cette zone est riche de 46 espèces ligneuses reparties en 22 familles et 38 genres. Les différents usages des ligneux révélés regroupent l'alimentation animale et humaine, la pharmacopée, l'artisanat, le bois-énergie et la construction. Cependant, l'étude à relevé que la surexploitation de ces ressources ligneuses présente des risques de disparition des espèces et compromette la régénération séminale de celles-ci. Les espèces ligneuses devraient alors bénéficier d'une attention particulière en vue de rationaliser leur utilisation. Il faudrait parallèlement former les villageois sur les techniques de prélèvement peu dommageable pour les espèces ligneuses. La pratique de la RNA et l'amorce de celle de la conservation des espèces observées dans la zone devraient être encouragées au même titre que les plantations des espèces indigènes.

\section{Remerciements}

Les auteurs tiennent à remercier vivement le Projet d'Appui à la Sécurité Alimentaire et au Développement dans la Région de Maradi (PASADEM) pour son appui financier dans la réalisation de ce travail, à travers la convention signée avec l'UDDM de Maradi.

\section{References:}

1. Abdourahamane, H., Morou, B., Rabiou, H. \& Mahamane, A. (2013). Caractéristiques floristiques, diversité et structure de la végétation ligneuse dans le Centre-Sud du Niger : cas du complexe des forêts classées de Dan kada Dodo-Dan Gado. Int. J. Biol. Chem. Sci., 7(3): 1048-1068.

2. Achigan-dako, E.G., Pasquini, M.W., Assogba, K.F., N'danikou, S., Dansi, A. \& Ambrose-oji, B. (2010). Traditional Vegetables in 
Benin. Institut National des Recherches Agricoles du Benin. Imprimerie du CENAP, Cotonou, 285p.

3. Adamou, S. (2012). Contribution à la capitalisation des bonnes pratiques agroforestières pour l'adaptation aux changements climatiques et l'atténuation des émissions de gaz à effet de serre au Niger. Mémoire de Master en Changement Climatique et Développement Durable, Agrhymet, 78p.

4. Anonyme, (2013). Plan du développement Communal (PDC). Commune Urbaine de Guidan Roumdji, Région de Maradi, 130p.

5. Baggnian, I. (2010). Rôle des dynamiques démographiques et migratoires sur l'évolution des écosystèmes sahéliens: cas d'un terroir villageois reverdi et non reverdi du département de Mirriah dans la région de Zinder au Niger. Mémoire de DESS en Protection de l'Environnement et Amélioration des Systèmes Agraires Sahéliens, Faculté d'Agronomie, Université Abdou Moumouni de Niamey, 77p.

6. Baggnian, I., Adamou M.M., Adam, T. \& Mahamane, A. (2013). Impact des modes de gestion de la Régénération Naturelle Assistée des ligneux (RNA) sur la résilience des écosystèmes dans le CentreSud du Niger. Journal of Applied Biosciences, 71:5742- 5752.

7. Boubacar, H. (2010). Caractérisation biophysique des ressources ligneuses dans les zones dégradées et reverdies au Sahel : cas de département de Mayahi. Mémoire DEA en biologie appliquée, Faculté des Sciences et Techniques, Université Abdou Moumouni de Niamey, 57p.

8. Dan Guimbo, I., Mahamane, A. \& Ambouta, J.M.K. (2010). Peuplement des parcs à Neocarya macrophylla (Sabine) Prance et à Vitellaria paradoxa (Gaertn. C.F.) dans le sud-ouest nigérien : diversité, structure et régénération. Int. J. Biol. Chem. Sci. 4(5): 17061720.

9. Dan Guimbo, I., Baragé, M. \& Douma S. (2012). Etudes préliminaires sur l'utilisation alimentaire des plantes spontanées dans les zones périphériques du parc W du Niger. Int. J. Biol. Chem. Sci., 6(6): 4007-4017.

10. Devineau, J.L. \& Guillaumet, J.L. (1992). Origine, nature et conservation des milieux naturels africains: le point de vue des botanistes. Afrique Contemporaine, 161: 79-90.

11. Dramé, Y.A. \& Berti, F. (2008). Les enjeux socio-économiques autour de l'agroforesterie villageoise à Aguié (Niger). Tropicultura, 26 (3):141-149. 
12. Fandohan, B. (2011). Conservation biology of Tamarindus indica (Fabaceae) in Benin, West Africa. PhD Thesis, University of Abomey-Calavi, Benin, 227p.

13. Fandohan, B., Glélé-Kakaï, R., Sinsin, B. \& Pelz D. (2008). Caractérisation dendrométrique et spatiale de trois essences ligneuses médicinales dans la forêt classée de Wari Maro au Bénin. Rev. Ivoir. Sci. Technol., 12:173 -186.

14. FAO, (2001). Produits forestiers non ligneux. Evaluation des ressources en produits forestiers non ligneux. Expérience et principe de biométrie. Rome, Italie, $117 \mathrm{p}$.

15. GERAD (Groupe d'Etude, de Recherche et d'Appui au Développement), 2010. Plan Local de Développement de la Communauté Rurale de Khossanto. Rapport, version finale, 74 pages.

16. Glèlè-Kakaï R., Assogbadjo A.E., Sinsin, B., Pelz D. (2009). Structure spatiale et régénération naturelle de Pterocarpus Erinaceus Poir. en zone soudanienne au Benin. Rev. Ivoir. Sci. Technol., 13: 199-212.

17. Guigma, Y., Zerbo, P. \& Millogo-Rasolodimby, J. (2012). Utilisation des espèces spontanées dans trois villages contigus du Sud du Burkina Faso. Tropicultura, 30(4) : 230-235.

18. Hamadou, H.Y. (2008). Etude floristique et ethnobotanique du terroir villageois de Boumba dans la réserve de biosphère du $\mathrm{W}$ du Niger. Mémoire d'Ingénieur de Techniques Agricoles (ITA), Faculté d'Agronomie, Université Abdou Moumouni de Niamey, 64p.

19. Inoussa, M.M. (2011). Dynamique des forêts claires dans le parc national du W du Niger. Thèse de Doctorat en Ecologie Végétale, Faculté des Sciences et Techniques, Université Abdou Moumouni. $138 \mathrm{p}$.

20. INS (Institut National de la Statistique), 2013. Projections démographiques de la population du Niger. Direction Nationale de la Statistique, Ministère du Plan, République du Niger, 10p.

21. Issa, Y. \& Oumarou, I. (2006). La crise alimentaire à Guidan Roumdji 2004-2005. Etudes et Travaux n ${ }^{\circ} 65$. Laboratoire d'Etudes et Recherches sur les Dynamiques Sociales et le Développement local (LASDEL), Niamey, Niger, 37p.

22. Kabore-Zoungrouna, C.Y. (1995). Composition chimique et valeur nutritive des herbacées et ligneux des pâturages naturels soudaniens et des sous-produits du Burkina-Faso, Thèse d'Etat, Université de Ouagadougou. 201p.

23. Larwanou, M., Abdoulaye, M. \& Reij, C. (2006). Etude de la régénération naturelle assistée dans la région de Zinder (Niger): 
première exploration d'un phénomène spectaculaire. International Ressources Group/USAID/Washington DC. 67p.

24. Mahamane, A. (2005). Études floristique, phytosociologique et phytogéographique de la végétation du Parc Régional du W du Niger. Thèse de doctorat en Sciences Agronomiques et Ingénierie Biologique, Faculté des Sciences, Université Libre de Bruxelles, 484p.

25. Natta, A.K., Yedomonhan, H., Zoumarou-wallis, N., Houndehin, J., Ewedje, E.B.K. \& Glèlé Kakai, R.L. (2011). Typologie et structure des populations naturelles de Pentadesma butyracea dans la zone soudano-guinéenne du Bénin. Annales des Sciences Agronomiques, 15 (2):137-152.

26. Ndiaye, M., Dione, M.E \& Akpo, L.E. (2010). Caractéristiques des ligneux dans les terroirs pastoraux de Ranérou (région de Matam, nord-Sénégal). J. Sci, 10(3): 12-27.

27. Ouédraogo, A., Thiombiano, A., Hahn-Hadjali, K. \& Guinko, S. (2005). Structure du peuplement juvénile et potentialités de régénération des ligneux dans l'Est du Burkina Faso. Etudes sur la flore et la végétation du Burkina Faso et des pays avoisinants. Sécheresse, 10: 17- 24.

28. Oumar, N.G., Oumar, S., Leonard, E.A. (2014). Richesse de la pharmacopée malinké : rôle médicinal de l'arbre a Khossanto : (Kédougou, Sénégal oriental). Journal of Applied Biosciences, 74: 6043- 6058 .

29. Oumorou, M., Sinadouwirou, T., Kiki, M., Glélé-Kakai, R., Mensah, G.A. \& Sinsin B. (2010). Disturbance and population structure of Vitex doniana $\mathrm{Sw}$. in northern Benin. Int. J. Biol.Chem.Sci., 4(3): 624-632.

30. Sani, R.A. (2009). Caractérisation biophysique des ressources ligneuses dans un site reverdi et un site dégradé dans le département de Mirriah. Mémoire de diplôme d'Ingénieur des Techniques et forestières, Faculté d'Agronomie, Université Abdou Moumouni de Niamey, 53p.

31. Sarr, O., Diatta, S., Gueye, M., Ndiaye, P.M., Guisse, A. \& Akpo, L.E. (2013). Importance des ligneux fourragers dans un système agropastoral au Sénégal (Afrique de l'ouest). Revue Méd. Vét., 164 (1): 2-8.

32. Savadogo, O.M., Ouattara, K., Pare, S., Ouedraogo, I., SawadogoKaboré, S., Barron, J. \& Zombre, N.P. (2016). Structure, composition spécifique et diversité des ligneux dans deux zones contrastées en zone Sahélienne du Burkina Faso. VertigO 16 (1), mis en ligne le 09 
mai 2016, consulté le 08 octobre 2016. URL : http://vertigo.revues.org/17282

33. Sinsin, B., Eyog-Matig, O., Assogbadjo, A.E., Gaoue, O. \& Sinadouwirou, T. (2004). Dendrometrics characteristics as indicators of pressure on Afzelia africana Sm. Dynamic changes in trees found in different climatic zones of Benin. Biodiversity and Conservation 13: $1555-1570$.

34. Thiombiano, D.N.E., Lamien, N., Dibong, D.S., Boussim, I.J. \& Belem, B. (2012). Le rôle des espèces ligneuses dans la gestion de la soudure alimentaire au Burkina Faso. Sécheresse; 23 : 86-93.

35. Tieguhong, J.C., Ndoye, O., Vantomme, P., Grouwels, S., Zwolinski, J. \& Masuch, J. (2009). Coping with crisis in Central Africa: enhanced role for non-wood forest products. Unasylva, 233(60):4954.

36. Yaméogo, J.T., Somé, A.N. \& Hien, M. (2009). Etude préliminaire à une restauration de sols dégradés en zone soudanienne du Burkina Faso. Sécheresse, 20(1): 8-32.

37. Zerbo, P., Rasolodimby, J.M., Ouedraogo O.G.N. \& Van Damme P., 2011. Plantes médicinales et pratiques médicales au Burkina Faso: cas des Sanan. Bois et forêts des tropiques, N 307 (1). 
Annexe 1. Etat des espèces ligneuses

\begin{tabular}{|c|c|c|c|c|c|}
\hline \multirow[b]{2}{*}{ Espèces ligneuses } & \multicolumn{5}{|c|}{ Etat } \\
\hline & Disparues & Menacées & Rares & \begin{tabular}{|l|} 
Introduites \\
\end{tabular} & Conservées \\
\hline Acacia erythrocalyx & $\mathrm{x}$ & & & & \\
\hline Acacia holosericea & & & & $\mathrm{x}$ & \\
\hline Acacia nilotica & & $\mathrm{x}$ & & & \\
\hline Acacia occidentale & $\mathrm{x}$ & & & & \\
\hline Acacia senegal & & $\mathrm{x}$ & & & \\
\hline Acacia seyal & & & $\mathrm{x}$ & & \\
\hline Acacia tortilis & & $\mathrm{x}$ & $\mathrm{x}$ & & \\
\hline Adansonia digitata & & & & & $\mathrm{x}$ \\
\hline Albizia chevalieri & & $\mathrm{x}$ & & & \\
\hline Anacardium occidentale & & & & $\mathrm{x}$ & \\
\hline Annona senegalensis & & $\mathrm{x}$ & & & \\
\hline Anogeissus leiocarpa & & $\mathrm{x}$ & & & \\
\hline Azadirachta indica & & & & & $\mathrm{x}$ \\
\hline Balanites aegyptiaca & & $\mathrm{x}$ & & & \\
\hline Bauhinia rufescens & & $\mathrm{x}$ & & & \\
\hline Bombax costatum & $\mathrm{x}$ & & & & \\
\hline Boscia angustifolia & $\mathrm{x}$ & & & & \\
\hline Boscia salicifolia & & & $\mathrm{x}$ & & \\
\hline Boscia senegalensis & & & $\mathrm{x}$ & & \\
\hline Boswellia dalzielii & & & $\mathrm{x}$ & & \\
\hline Calotropis procera & & & $\mathrm{x}$ & & \\
\hline Capparis corymbosa & $\mathrm{x}$ & & & & \\
\hline Cassia sangueana & & $\mathrm{x}$ & & & \\
\hline Cassia sieberiana & & & $\mathrm{x}$ & & \\
\hline Ceiba pentandra & $\mathrm{x}$ & & & & \\
\hline Combretum glutinosum & & & & & $\mathrm{x}$ \\
\hline Combretum micranthum & & $\mathrm{x}$ & & & \\
\hline Combretum nigricans & $\mathrm{x}$ & & & & \\
\hline Commiphora africana & & $\mathrm{x}$ & & & \\
\hline Cordia sinensis & $\mathrm{x}$ & & & & \\
\hline Crateva religiosa & $\mathrm{x}$ & & & & \\
\hline Detarium microcarpum & & & & $\mathrm{x}$ & \\
\hline Dichrostachys cinerea & & $\mathrm{x}$ & & & \\
\hline Diospyros mespiliformis & & & $\mathrm{x}$ & & \\
\hline Entada africana & & & $\mathrm{x}$ & & \\
\hline Eucalyptus camaldulensis & & & & $\mathrm{x}$ & \\
\hline Faidherbia albida & & & & & $\mathrm{x}$ \\
\hline Feretia apodanthera & & $\mathrm{x}$ & & & \\
\hline Ficus dekdekena & $\mathrm{x}$ & & & & \\
\hline Ficus platyphylla & & & $\mathrm{X}$ & & \\
\hline Ficus polita & & $\mathrm{x}$ & & & \\
\hline Ficus thonningii & $\mathrm{x}$ & & & & \\
\hline Gardenia sokotensis & $\mathrm{x}$ & & & & \\
\hline Grewia bicolor & & & $\mathrm{x}$ & & \\
\hline
\end{tabular}




\begin{tabular}{|c|c|c|c|c|c|}
\hline Grewia flavescens & & & $\mathrm{x}$ & & \\
\hline Grewia tenax & $\mathrm{x}$ & & & & \\
\hline Guiera senegalensis & & $\mathrm{x}$ & & & \\
\hline Hyphaene thebaica & & & $\mathrm{x}$ & & \\
\hline Isoberlinia doka & $\mathrm{x}$ & & & & \\
\hline Khaya senegalensis & $\mathrm{x}$ & & & & \\
\hline Lannea microcarpa & & & & & $\mathrm{x}$ \\
\hline Lannea schimperi & $\mathrm{x}$ & & & & \\
\hline Maerua angolensis & & & $\mathrm{x}$ & & \\
\hline Maerua crassifolia & & $\mathrm{x}$ & & & \\
\hline Mangifera indica & & & & $\mathrm{x}$ & \\
\hline Moringa oleifera & & & & $\mathrm{x}$ & \\
\hline Neocarya macrophylla & & & & $\mathrm{x}$ & \\
\hline Parkia biglobosa & & & & $\mathrm{x}$ & \\
\hline Parkinsonia acculeata & $\mathrm{x}$ & & & & \\
\hline Pericopsis laxiflora & $\mathrm{x}$ & & & & \\
\hline Phyllantus muellerianus & $\mathrm{x}$ & & & & \\
\hline Piliostigma reticulatum & & & & & \\
\hline Prosopis africana & & $\mathrm{x}$ & & & \\
\hline Prosopis juliflora & & & & $\mathrm{x}$ & \\
\hline Pterocarpus erinaceus & $\mathrm{x}$ & & & & \\
\hline Sclerocarya birrea & & & & & \\
\hline Securidaca & $\mathrm{x}$ & & & & \\
\hline longepedunculata & & & $\mathrm{x}$ & & \\
\hline Sterculia setigera & & & & & \\
\hline Stereospermum kunthianum & & $\mathrm{x}$ & & & \\
\hline Strychnos innocua & & & & & \\
\hline Terminalia avicennioides & & & & & \\
\hline Terminalia mantaly & & & & & \\
\hline Vitellaria paradoxa & & & & & \\
\hline Vitex doniana & & & & & \\
\hline Ximenia americana & $\mathrm{x}$ & & & & \\
\hline Xeromphis nilotica & $\mathrm{x}$ & & & & \\
\hline Ziziphus mucronata & $\mathrm{x}$ & & & \\
\hline Ziziphus spina-christi & $\mathrm{x}$ & & & & \\
\hline
\end{tabular}

